\title{
The Effect of the Electron Temperature on Incompressible Strips in Quantum Hall Regime
}

\author{
N. Boz YurdaşAN ${ }^{a}$, A. SidDiki ${ }^{b}$ AND İ. SÖKMEN ${ }^{a}$ \\ ${ }^{a}$ Dokuz Eylül University, Faculty of Sciences, Physics Department, Tinaztepe Campus, 35160, Izmir, Turkey \\ ${ }^{b}$ Istanbul University, Physics Department, Faculty of Sciences, 34134-Vezneciler-Istanbul, Turkey \\ We used a theory of thermo-hydrodynamics in quantum Hall system observed on a two-dimensional system in \\ high magnetic fields at low temperatures, to investigate the electron temperature in the linear response regime. The \\ variation of electron temperature exhibits an antisymmetric distribution of the incompressible strips. According \\ to this result, we obtain effects of the electron temperature on the current density distribution using a Thomas- \\ Fermi-Poisson approximation. We observe that incompressible strips change with increasing and/or decreasing the \\ electron temperature with regard to the lattice temperature.
}

DOI: 10.12693/APhysPolA.123.314

PACS: 73.43.-f, 73.43.Cd, 44.30.+v, 31.15.B-

\section{Introduction}

Apart from sample properties, such as electron density, mobility and sample geometry, the temperature of the sample and the electrical current flow through it are important aspects of the integer quantum Hall effect. In this effect, the Hall resistance $R_{\mathrm{H}}$ of the $i$-th plateau of a fully-quantized two-dimensional electron system (2DES) has the value $R_{\mathrm{H}}(i)=h /\left(i e^{2}\right)$, where $h$ is the Planck constant, $e$ is the elementary charge, and $i$ is an integer $[1,2]$. The physical quantities, as the temperature, the strength of the magnetic field and the imposed current determine the existence of incompressible strip (IS) that carries the imposed current in a quantized number of levels. The width and the position of IS are closely related to these physical dependences $[3,4]$.

In our previous work, we calculated the variation of the electron temperature in the linear response regime, employing the thermo-hydrodynamic theory in quantum Hall systems (QHS). The theory of thermo-hydrodynamics is bound by conservation of electron number and thermal flux densities, following Akera and his co-workers $[5,6]$. We imposed realistic boundary conditions and therefore described experimental systems accurately. We observed that the electron temperature deviation oscillates as a function of the lattice temperature in the ISs. According to this result, in this study we investigate the current density profile of $2 \mathrm{DES}$ in the presence of an electron temperature using the self-consistent Thomas-Fermi-Poisson approximation (TFPA) [7]. The current density is calculated from a local version of the Ohm law. This theoretical work confirms the experimental evidence and shows that the current flows in the ISs, in which the local longitudinal resistivity vanishes. Another result shows that the effect of electron temperature deviation on the current density distribution is so strong, that ISs change completely according to the ISs obtained at a constant lattice temperature.

\section{Model}

Following previous works, we consider the Hall bar as a 2DES in the $x-y$ plane that is in a perpendicular magnetic field $\boldsymbol{B}=(0,0, B)[7,8]$. The electron density $n_{\mathrm{el}}(y)$ are confined by the confinement potential $V_{\mathrm{bg}}(y)$ due to donors which are distributed uniformly in the sample. To describe the experimental geometries, we impose boundary conditions such that two metallic gates reside at the physical edges, following Chklovskii et al. The effective potential within the semi-classical approximation is

$$
V(y)=V_{\mathrm{bg}}(y)+V_{\mathrm{H}}(y)
$$

with the confinement potential and the Hartree potential

$$
\begin{aligned}
& V_{\mathrm{bg}}(y)=-E_{\mathrm{bg}}^{0} \sqrt{1-\left(\frac{y}{d}\right)^{2}}, \\
& V_{\mathrm{H}}(y)=\frac{2 e^{2}}{\kappa} \int_{-d}^{d} \mathrm{~d} y^{\prime} K\left(y y^{\prime}\right) n_{\mathrm{el}}\left(y^{\prime}\right) .
\end{aligned}
$$

Here $E_{\mathrm{bg}}^{0}$ is the pinch-off energy which defines the minimum of the bare confinement potential [9], $\kappa$ is the dielectric constant, $n_{0}$ is the donor density and $2 d$ is the sample width. The Hartree potential due to the 2DES is determined via Poisson's equation by the electron density $n_{\mathrm{el}}(y)$. Kernel $K\left(y, y^{\prime}\right)$ solves Poisson's equation under the given boundary conditions [8]. The electron density is, in turn, determined by the effective potential $V(y)$ and calculated in the Thomas-Fermi approximation

$$
n_{\mathrm{el}}(y)=\int \mathrm{d} E D(E) f\left(E+V(y)-\mu_{\mathrm{ec}}\right),
$$

with $D(E)$ - the density of states (DOS), $f(E)=$ $1 /\left[\exp \left(\left(\varepsilon-\mu_{\mathrm{ec}}\right) / k_{\mathrm{B}} T_{\mathrm{e}}\right)+1\right]$ - the Fermi function, $k_{\mathrm{B}}$ - the Boltzmann constant, $T_{\mathrm{e}}$ - the electron temperature and $\mu_{\mathrm{ec}}$ - the constant electrochemical potential in the equilibrium state $[8,10]$.

In this work, we apply the local equilibrium approximation, used extensively in many systems [11]. In local equilibrium, the lattice temperature $T_{\mathrm{L}}$ remains unchanged by the presence of the applied current. In the 
presence of a dissipative current $I=\int_{-d}^{d} \mathrm{~d} y j_{n_{\mathrm{el}} x}(y)$, the electrochemical potential $\mu_{\mathrm{ec}}(\boldsymbol{r})$ depends on position and its gradient $\boldsymbol{E}=\nabla \mu_{\mathrm{ec}}(\boldsymbol{r}) / e$ satisfies the local Ohm law $\hat{\rho}(\boldsymbol{r}) \boldsymbol{j}_{n_{\mathrm{el}}}(\boldsymbol{r})=\boldsymbol{E}(\boldsymbol{r})$ with resistivity tensor.

In the previous work, we employed the theory of thermo-hydrodynamics described by equations of conservation with electron number and thermal flux densities in QHS to obtain the variations of the electron temperature in the linear-response regime [12]. We consider two hydrodynamic equations and assume that the electron number and the total energy of the system are conserved. The equations of electron number conservation and energy conservation are formulated by

$$
\begin{aligned}
& \frac{\partial n_{\mathrm{el}}}{\partial t}=-\nabla \cdot \boldsymbol{j}_{n_{\mathrm{el}}}, \\
& \frac{\partial \varepsilon}{\partial t}=-\nabla \cdot \boldsymbol{j}_{\varepsilon}-P_{\mathrm{L}},
\end{aligned}
$$

respectively. Here $\boldsymbol{j}_{n_{\mathrm{el}}}$ is the number flux density, $\boldsymbol{j}_{\varepsilon}$ is the energy flux density and $P_{\mathrm{L}}$ is the energy loss per unit area due to the heat transfer between electrons and phonons [11]. The time evolution of the entropy density $s$ is derived by using Eqs. (4), (5), and by the fundamental thermodynamical equation

$$
T_{\mathrm{e}} \frac{\partial s}{\partial t}=-\nabla \cdot \boldsymbol{j}_{q}-\nabla \mu_{\mathrm{ec}} \cdot \boldsymbol{j}_{n_{\mathrm{el}}}-P_{\mathrm{L}},
$$

where the thermal flux density $\boldsymbol{j}_{q}$ is described by

$$
\boldsymbol{j}_{q}=\boldsymbol{j}_{\varepsilon}-\mu_{\mathrm{ec}} \boldsymbol{j}_{n_{\mathrm{el}}} .
$$

We assume translation invariance in the $x$ direction. Therefore the electron temperature $T_{\mathrm{e}}$ and the chemical potential $\mu$ are independent of $x$ direction. Also electric field $E_{x}$ in $x$ direction becomes $e E_{x}=\nabla_{x}(\Delta V)$ because of $\nabla_{x}(\Delta \mu)=0$. After these conditions two equations of conservation become

$$
\begin{aligned}
& \Delta j_{n_{\mathrm{el}}}(y)=0 \quad(|y|<d), \\
& \nabla_{y}\left(\Delta j_{q}(y)\right)+e E_{x} j_{n_{\mathrm{el}}}(y)+P_{\mathrm{L}}=0,
\end{aligned}
$$

with the deviations from the equilibrium values [13]. In the linear response regime the energy loss $P_{\mathrm{L}}$ is written as $P_{\mathrm{L}}=C_{p}\left(T_{\mathrm{e}}-T_{\mathrm{L}}\right)$. Here,

$$
C_{p}=C_{p}^{0} \int \rho(\varepsilon)\left(-\frac{\partial f\left(\varepsilon_{N}, \mu_{\mathrm{ec}}, T_{\mathrm{e}}\right)}{\partial \varepsilon}\right) \mathrm{d} \varepsilon
$$

includes the density of state $\rho(\varepsilon)$ written as

$$
\rho(\varepsilon)=\frac{1}{\pi l^{2}} \sum_{N} \frac{1}{\sqrt{\pi} \Gamma_{\mathrm{L}}} \exp \left(-\frac{\left(\varepsilon_{N}-\varepsilon\right)^{2}}{\Gamma_{\mathrm{L}}^{2}}\right)
$$

with the Landau level broadening $\Gamma_{\mathrm{L}}$. The coefficient $C_{p}^{0}$ is defined by the transition rate due to electron phonon scattering and is estimated to be $1.4 \times 10^{-5} k_{\mathrm{B}} E_{0}^{2} / \hbar$ from the experimental results by Komiyama et al. [14].

\section{Results and discussion}

Figure 1 presents the calculated results of the electron temperature for several values of coefficients, $C_{p}^{0}=$ $3 \times 10^{-2}, 5 \times 10^{-2}$ and $8 \times 10^{-2}$ at (a) $\nu(0)=2.0$ and $(\mathrm{b})$ $\nu(0)=2.5$. The lattice temperature is $T_{\mathrm{L}}=0.15 E_{\mathrm{F}}^{0} / k_{\mathrm{B}}$ with the Fermi energy at the center $E_{\mathrm{F}}^{0}=9.615 \mathrm{meV}$.
The variation of the electron temperature without electron phonon interaction is larger than the variation of the electron temperature with the interactions, since a part of the energy is converted to the heat energy between electrons and phonons because of the interaction.

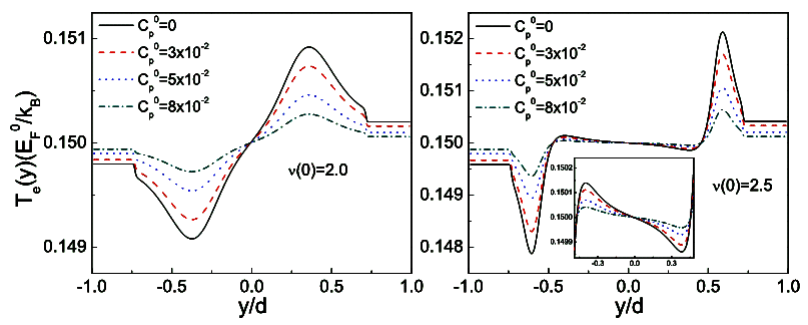

Fig. 1. The electron temperature versus position, calculated for different $\nu(0)$ at fixed lattice temperature $T_{\mathrm{L}}=0.15 E_{\mathrm{F}}^{0} / k_{\mathrm{B}}$. The sample parameters are width $2 d=2.2 \mu \mathrm{m}$, the density of donor $n_{0}=3.6 \times 10^{11} \mathrm{~cm}^{-2}$ and the Fermi energy $E_{\mathrm{F}}^{0}=9.615 \mathrm{meV}$ corresponding to the electron density at the center. The inset shows the enlarged electron temperature for the center of the sample.

We show the changes in the electron temperature $T_{\mathrm{e}}$ with increasing the filling factor at fixed lattice temperature $T_{\mathrm{L}}=0.03 E_{\mathrm{F}}^{0} / k_{\mathrm{B}}$ in Fig. 2 . This figure shows that the electron temperature oscillates as a function of the lattice temperature in the ISs. Also it is shown that the electron temperature strongly depends on the filling factor. Since ISs are inversely proportional to the filling factor and approach the sample edge, the ISs become smaller slowly and move towards the sample edges with increasing the filling factor.

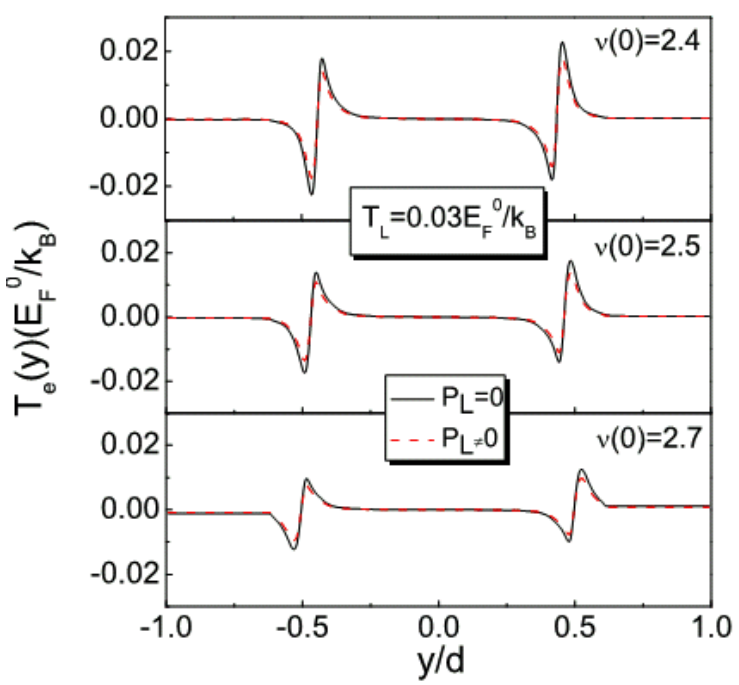

Fig. 2. The electron temperature versus position, calculated for $\nu(0)=2.4,2.5$ and 2.7 at fixed lattice temperature $T_{\mathrm{L}}=0.03 E_{\mathrm{F}}^{0} / k_{\mathrm{B}}$. The solid and dash lines represent the results for without energy loss and with energy loss, respectively. 
In Fig. 3a, the electron temperature and lattice temperature are taken into account to obtain the current density profile. When the lattice temperature is taken into account, ISs on both sides of the sample are the same since $T_{\mathrm{L}}$ is uniform in the system. However in the presence of electron temperature, left side of the sample heats up and the right side of the sample is cooled down. Therefore, interestingly, ISs show different behavior. This effect is more evident at $\nu(0)=2.4$, so that the results are shown for $\nu(0)=2.4$. As we know, the widths of ISs increase monotonically with decreasing temperature. The result shows that when the electron temperature becomes smaller than the lattice temperature (left side), the 2DES develops ISs with low longitudinal resistivity and the current density is increasingly confined to the ISs. Therefore, the dash peak is larger than the solid peak on the left hand side. On the other hand, the other side of the sample (right side) is opposite.

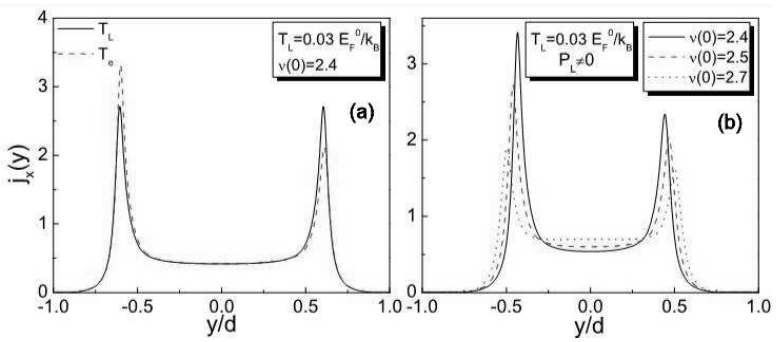

Fig. 3. The current density versus position for (a) $\nu(0)=2.4$ calculated within the lattice temperature and electron temperature, (b) $\nu(0)=2.4,2.5$ and 2.7 calculated within electron temperature at fixed lattice temperature $T_{\mathrm{L}}=0.03 E_{\mathrm{F}}^{0} / k_{\mathrm{B}}, P_{\mathrm{L}} \neq 0$.

In Fig. 3b, we consider the energy loss $P_{\mathrm{L}}$ to investigate the current density profile using the electron temperature. As expected, with increasing the center of the filling factor $\nu(0)$, the widths of ISs become smaller and move from the center towards the edges. The electron temperature becomes larger than the lattice temperature on the right hand side. Therefore IS at the right side becomes narrow for each $\nu(0)$ depending on the electron temperature. Since the variation of electron temperature is much bigger, this difference is larger at $\nu(0)=2.4$, as is seen in Fig. 2. The variation of the electron temperature becomes smaller at $\nu(0)=2.7$, therefore the difference between two ISs nearly disappears.

In summary, we calculate the current density of $2 \mathrm{DES}$ in the presence of the electron temperature using the self-consistent TFPA. We observe that the widths of the ISs depend strongly on the spatial variation of the electron temperature. ISs change with increase and/or decrease of the electron temperature with regard to the lattice temperature. Also with increase of the filling factor, the deviation of the electron temperature decreases. Therefore the width of ISs becomes smaller and moves from the center towards the sample edges.

\section{References}

[1] K. v. Klitzing, G. Dorda, M. Pepper, Phys. Rev. Lett. 45, 494 (1980)

[2] D.C. Tsui, H.L. Stormer, A.C. Gossard, Phys. Rev. Lett. 48, 1559 (1982)

[3] A. Siddiki, R.R. Gerhardts Phys. Rev. B 70, 195335 (2004).

[4] A. Siddiki, R.R. Gerhardts, Int. J. Mod. Phys. B 21, 1362 (2007)

[5] H. Akera, J. Phys. Soc. Jpn. 69, 3174 (2000).

[6] H. Akera, J. Phys. Soc. Jpn. 70, 1468 (2001).

[7] A. Siddiki, R.R. Gerhardts, Phys. Rev. B 68, 25315 (2003).

[8] K. Güven, R.R. Gerhardts, Phys. Rev. B 67, 115327 (2003).

[9] A. Siddiki, S. Kraus, R.R. Gerhardts, Physica E 34, 136 (2006)

[10] R.R. Gerhardts, Phys. Status Solidi B 245, 378 (2008)

[11] H. Akera, H. Suzuura, J. Phys. Soc. Jpn. 74, 997 (2005)

[12] N. Boz Yurdasan, K. Akgüngör, A. Siddiki, İ. Sökmen, Physica E 44, 1367 (2012)

[13] S. Kanamaru, H. Suzuura, H. Akera, J. Phys. Soc. Jpn. 75, 064701 (2006)

[14] S. Komiyama, T. Takamasu, S. Hiyamizu, S. Sasa, Solid State Commun. 54, 479 (1985). 